\title{
Formação de profissionais de Educação Física que atuam nos núcleos de apoio à saúde da família do Estado de São Paulo
}

http://dx.doi.org/10.11606/1807-5509201800040647

\author{
Alexandre ROMERO* \\ Paulo Henrique GUERRA* \\ Alex FLORINDO*
}

\author{
${ }^{*}$ Escola de \\ Artes, Ciências \\ e Humanidades, \\ Universidade de São \\ Paulo, São Paulo, SP,
}

\section{Resumo}

0 objetivo do presente estudo foi descrever aspectos relacionados à formação superior de profissionais de Educação Física (PEF) atuantes nos Núcleos de Apoio à Saúde da Família do estado de São Paulo (NASF). Para tanto, se conduziu um inquérito transversal descritivo, por meio de entrevista estruturada via telefone, no qual foram coletados dados sobre a formação de 91 PEF vinculados aos NASF. Como resultados, observou-se que apenas 22\% dos PEF tiveram contato com o tema "Saúde Pública/Saúde Coletiva" no curso de graduação $(n=20)$ e menos de $5 \%$ fizeram residência multiprofissional em saúde $(n=5)$. A maioria dos profissionais $(61,5 \%, n=56)$ discordou que teve formação adequada para atuar no SUS, e este percentual de discordância não teve grandes alterações segundo a faixa etária, tempo de conclusão de curso de graduação e se fez ou não curso de pós-graduação. Os dados apresentados mostram que os PEF atuantes nos NASF do estado de São Paulo relataram que tiveram uma formação distanciada daquela que se pretende para a atuação neste contexto. Reflexões e discussões sobre possiveis melhoras na formação do curso de graduação e de pós-graduação dos PEF para atuarem nos NASF devem ser fomentadas por gestores e professores que atuam nesta área.

Palavras-chave: Sistema Único de Saúde; Educação Física; Formação Profissional; Saúde Pública.

\section{Introdução}

A introdução do Sistema Único de Saúde (SUS) a partir da Constituição Federal de 1988, representou o rompimento do Ministério da Saúde brasileiro para com o modelo de saúde biomédico centrado na cura da doença. Após sua regulamentação, em 1990, passou a vigorar no país um modelo de sistema de saúde socialmente construído ${ }^{1}$, que leva em consideração os aspectos sociais, ambientais, políticos e culturais, valorizando açóes de promoção, proteção, recuperação e manutenção da saúde individual e coletiva ${ }^{2-4}$.

$\mathrm{Na}$ perspectiva do SUS, o foco das estratégias volta-se à promoção da saúde, buscando envolver parcerias permanentes do Estado, da comunidade, de indivíduos, e de outros órgãos setoriais, no sentido de promover e estreitar ações intersetoriais ${ }^{4}$. Nesta trajetória, a implantação da Política Nacional de Promoção da Saúde foi um marco ${ }^{3}$ e envolve a promoção da atividade física 5 .

Entretanto, à parte do gradual reconhecimento e disseminação dos benefícios à saúde que estão associados diretamente à prática regular de atividades físicas moderadas e vigorosas, suas prevalências ainda são baixas na população adulta brasileira, principalmente no tempo livre (lazer) e como forma de deslocamento $(14,8 \%$ e $11,7 \%$, respectivamente $)^{6,7}$. Na tentativa de mudança deste cenário, a promoção da atividade física tem sido posta como uma das principais estratégias de ação no contexto do SUS. Como elementos deste esforço contínuo ressaltam-se a criação dos Núcleos de Apoio à Saúde da Família (NASF) em 2008 e o Programa Academia da Saúde ${ }^{8,9}$.

Os Profissionais de Educação Física (PEF) desempenham um importante papel neste contexto, por sua possível inserção enquanto agentes qualificadores das açóes interdisciplinares em saúde que envolvem a promoção da atividade física. Estudo recente realizado com amostra de municípios que foram contemplados com recursos do Ministério da Saúde para o desenvolvimento do Programa Academia 
da Saúde mostrou que municípios que contavam com PEF em equipes de NASF desenvolviam um número maior de estratégias de promoção da atividade física em comparaçáo com aqueles que náo contavam ${ }^{10}$.

Mesmo com a importância e o potencial de ação dos PEF na promoção da atividade física no contexto do
SUS, pouco se conhece sobre sua formação no curso de graduação e na pós-graduação e sua opinião sobre a preparação profissional para atuaçáo no SUS e NASF.

Partindo desta lacuna, o objetivo do presente estudo foi descrever aspectos relacionados à formação superior de PEF que atuam nos NASF do Estado de Sáo Paulo.

\section{Método}

\section{Visão geral}

Este foi um inquérito transversal com entrevista estruturada aplicada via telefone e todo o detalhamento desta aplicaçẫo e do questionário está descrito no estudo publicado por Romero et al. ${ }^{11}$. De forma resumida, o inquérito foi conduzido entre julho de 2013 e fevereiro de 2014 e envolveu 91 PEF que atuavam há pelo menos três meses nos NASF do Estado de São Paulo. A maioria dos profissionais tinha idade até 34 anos (54.9\%), a média de idade foi de 34,3 anos, eram homens $(54.9 \%)$ e até 10 anos de formação de graduação $(71.4 \%)^{11}$.

\section{Variáveis estudadas}

Com o objetivo de descrever as características de formação profissional dos PEF atuantes nos NASF do estado de São Paulo, foram avaliadas as seguintes variáveis: tempo de atuação no NASF; tipo de instituição de ensino superior (IES) em que se formou; contato com o tema "Saúde Pública/Saúde Coletiva", realização de pós-graduação na área da saúde e grau de concordância sobre formação adequada para atuação profissional na área de "Saúde Pública/Saúde Coletiva".

\section{Análises de dados}

Todos os dados coletados foram digitados duplamente no programa Epi-Data versão $3.1 \mathrm{e}$ conferidos no módulo Validate para a correção das inconsistências. Foram feitas análises descritivas por meio de médias e análises de frequência (\%). Para realização de todas as análises foi utilizado o programa SPSS versão 15.0.

\section{Aspectos éticos}

Esta pesquisa foi aprovada pelo Comitê de Ética em Pesquisa da Escola de Artes, Ciências e Humanidades da Universidade de São Paulo (protocolo no 294.876) e pelo Comitê de Ética em Pesquisada da Secretaria Municipal da Saúde de São Paulo (parecer no 34/13).

\section{Resultados}

A TABELA 1 aponta que a maioria dos PEF entrevistados atua nos NASF há menos de quatro anos $(\mathrm{n}=82 ; 90,1 \%)$ e que, por tipo de IES, a maior parte dos PEF é egressa de instituiçôes privadas $(\mathrm{n}=79 ; 86,8 \%)$.

A TABELA 2 mostra que foi baixa a proporção de entrevistados $(n=20 ; 22 \%)$ que tiveram algum contato com o tema "Saúde Pública/Saúde Coletiva" durante o curso de graduação e também que foi muito baixa a proporção de respondentes que realizaram estágio(s) supervisionado(s) na temática "Saúde Pública/Saúde Coletiva" $(\mathrm{n}=5 ; 5,5 \%)$. Complementarmente, também se aponta na TABELA 2 que apenas 33 PEF responderam positivo sobre a realização de pósgraduação na área da saúde (36,3\%).
Dos 20 PEF que tiveram contato com o tema "Saúde Pública/Saúde Coletiva" no curso de graduação, a maioria relatou que foi por meio de disciplinas específicas $(\mathrm{n}=12 ; 60 \%)$ (FIGURA 1 ).

A TABELA 3 mostra que a maioria dos profissionais discordou que seu curso de graduação em Educaçáo Física ofereceu uma formação adequada para atuação profissional na área de "Saúde Pública/ Saúde Coletiva” ( $\mathrm{n}=56,61,5 \%)$.

Complementarmente, os dados estratificados por idade, tempo de conclusão do curso de graduaçáo em Educação Física (anos) e diploma de pós-graduação também apontam que a maioria dos PEF discordou que seu curso de Educação Física ofereceu uma formação adequada para atuação profissional na área de "Saúde Pública/Saúde Coletiva" (TABELA 3). 
TABELA 1 - Tempo de atuação nos Núcleos de Apoio à Saúde da Família e tipo de instituição de ensino superior em que os respondentes se formaram $(n=91)$

\begin{tabular}{lcc}
\hline Variáveis & $\mathbf{n}$ & $\mathbf{\%}$ \\
\hline Tempo de atuação no NASF & & \\
\hline$<2$ anos & 27 & 29,7 \\
2 a 4 anos & 55 & 60,4 \\
$>4$ anos & 9 & 9,9 \\
\hline Tipo de IES em que se formou & 12 & 13,2 \\
\hline Pública & 79 & 86,8 \\
Privada & 79 \\
\hline
\end{tabular}

TABELA 2 - Aspectos da formação dos Profissionais de Educação Física que atuavam nos Núcleos de Apoio à Saúde da Família do Estado de São Paulo, segundo contato com disciplinas e estágios e formação continuada relacionados com o tema, Agosto de 2013

\begin{tabular}{lcc}
\hline Variáveis & $\mathbf{n}$ & $\%$ \\
\hline Contato com o tema "Saúde Coletiva/ Saúde Pública” no curso de graduação & & \\
\hline Não & 71 & 78 \\
Sim & 20 & 22 \\
\hline Realização de estágio(s) supervisionado(s) na temática "Saúde Pública/Saúde Coletiva” & \\
\hline Não & 86 & 94,5 \\
Sim & 5 & 5,5 \\
\hline Realização de pós-graduação na área da saúde & & \\
\hline Não & 58 & 63,7 \\
Sim & 33 \\
\hline
\end{tabular}

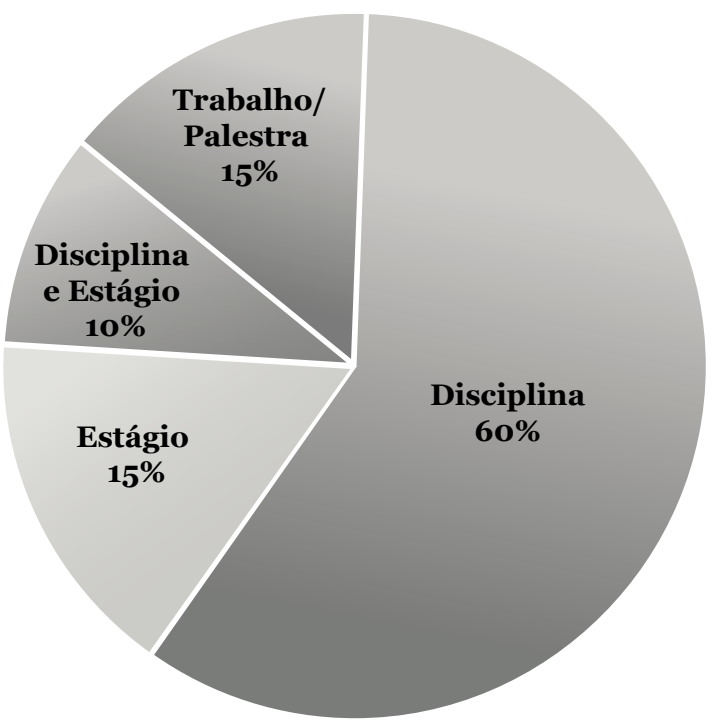

FIGURA 1 - Forma de Contato com o tema "Saúde Coletiva/ Saúde Pública” no curso de graduação $(\mathrm{n}=20)^{*}$. *: Pergunta direcionada aos 20 profissionais que responderam sim na questão "Contato com o tema 'Saúde Coletiva/Saúde Pública' no curso de graduação”. 
TABELA 3 - Opinião sobre a formação para atuação profissional na área de Saúde Pública/Saúde Coletiva geral e estratificado por faixa etária e de aspectos de formação de graduação e de pós-graduação dos Profissionais de Educação Física que atuavam nos Núcleos de Apoio à Saúde da Família do Estado de São Paulo, agosto de 2013.

\begin{tabular}{|c|c|c|}
\hline Variáveis & $\mathbf{n}$ & $\%$ \\
\hline \multicolumn{3}{|c|}{ Grau de concordância sobre formação adequada para atuaçáo profissional na área de Saúde } \\
\hline \multicolumn{3}{|l|}{ Pública/Saúde Coletiva } \\
\hline Concordaram & 23 & 25,3 \\
\hline Nem concordaram nem discordaram & 12 & 13,2 \\
\hline Discordaram & 56 & 61,5 \\
\hline \multicolumn{3}{|l|}{ Idade } \\
\hline \multicolumn{3}{|l|}{$\leq 34 \operatorname{anos}(n=55)$} \\
\hline Concordaram & 15 & 27,3 \\
\hline Nem concordaram nem discordaram & 6 & 10,9 \\
\hline Discordaram & 34 & 61,8 \\
\hline \multicolumn{3}{|l|}{$>34 \operatorname{anos}(n=36)$} \\
\hline Concordaram & 8 & 22,2 \\
\hline Nem concordaram nem discordaram & 6 & 16,7 \\
\hline Discordaram & 22 & 61,1 \\
\hline \multicolumn{3}{|c|}{ Tempo de conclusáo do curso de graduaçáo em Educaçáo Física (anos) } \\
\hline \multicolumn{3}{|l|}{$\leq 5 \operatorname{anos}(n=26)$} \\
\hline Concordaram & 7 & 26,9 \\
\hline Nem concordaram nem discordaram & 3 & 11,5 \\
\hline Discordaram & 16 & 61,6 \\
\hline \multicolumn{3}{|l|}{$6-10$ anos $(n=39)$} \\
\hline Concordaram & 9 & 23,1 \\
\hline Nem concordaram nem discordaram & 7 & 17,9 \\
\hline Discordaram & 23 & 59,0 \\
\hline \multicolumn{3}{|l|}{$>10$ anos $(n=26)$} \\
\hline Concordaram & 7 & 26,9 \\
\hline Nem concordaram nem discordaram & 2 & 7,7 \\
\hline Discordaram & 17 & 65,4 \\
\hline \multicolumn{3}{|c|}{ Modalidades de cursos de graduaçáo em Educaçáo Física } \\
\hline \multicolumn{3}{|l|}{ Licenciatura Plena $(n=49)$} \\
\hline Concordaram & 13 & 26,5 \\
\hline Nem concordaram nem discordaram & 4 & 8,2 \\
\hline Discordaram & 32 & 65,3 \\
\hline \multicolumn{3}{|l|}{ Bacharelado $(n=11)$} \\
\hline Concordaram & 3 & 27,3 \\
\hline Nem concordaram nem discordaram & 2 & 18,2 \\
\hline Discordaram & 6 & 54,5 \\
\hline \multicolumn{3}{|l|}{ Licenciatura e Bacharelado $(n=31)$} \\
\hline Concordaram & 7 & 22,6 \\
\hline Nem concordaram nem discordaram & 6 & 19,4 \\
\hline Discordaram & 18 & 58,0 \\
\hline
\end{tabular}


Continuação

TABELA 3 - Opinião sobre a formação para atuação profissional na área de Saúde Pública/Saúde Coletiva geral e estratificado por faixa etária e de aspectos de formação de graduação e de pós-graduação dos Profissionais de Educação Física que atuavam nos Núcleos de Apoio à Saúde da Família do Estado de São Paulo, agosto de 2013.

\begin{tabular}{lcc}
\hline Variáveis & n & \% \\
\hline Diploma de pós-graduaçáo & & \\
\hline Possui diploma de pós-graduação na área da saúde $(n=33)$ & 9 & 27,3 \\
\hline Concordaram & 5 & 15,1 \\
Nem concordaram nem discordaram & 19 & 57,6 \\
Discordaram & & \\
\hline Não possui diploma de pós-graduação na área da saúde $(n=58)$ & 14 & 24,1 \\
\hline Concordaram & 7 & 12,1 \\
Nem concordaram nem discordaram & 37 & 63,8 \\
Discordaram & &
\end{tabular}

\section{Discussão}

O presente trabalho teve como objetivo principal descrever a opiniáo dos PEF que atuam nos NASF do Estado de São Paulo sobre sua formação para atuação na área de Saúde Pública/Saúde Coletiva. Como principais achados, observou-se que a maioria dos PEF teve pequeno contato com o tema "Saúde Pública/Saúde Coletiva" durante o curso de graduação e a maioria discordou que sua formaçáo em Educação Física foi adequada para atuação profissional na área de "Saúde Pública/Saúde Coletiva”.

A respeito da minoria de PEF que tiveram contato com disciplinas específicas relacionadas à "Saúde Pública/Saúde Coletiva” ao longo do curso de graduação, as evidências disponíveis nos artigos de Costa et al..$^{12}$, Scabar ${ }^{13}$ e Anjos e Duarte ${ }^{14}$ podem responder, em boa parte, a esta questáo, uma vez que apontam a inexistência de disciplina específicas referentes à área da "Saúde Pública/Saúde Coletiva" em matrizes curriculares de diversos cursos de graduaçáo em Educação Física oferecidos no país.

Além disso, tanto no estudo de Anjos e Duarte ${ }^{14}$, quanto no estudo de Souza e Loch ${ }^{15}$, verificou-se que quando os cursos de EF continham disciplinas relacionadas ao campo da saúde, as mesmas apresentavam uma abordagem curativa e de prescrição. Para Anjos e Duarte ${ }^{14}$, as disciplinas específicas que abordam a "Saúde Pública/Saúde Coletiva" sáo de grande importância, pois, além da preparaçáo para o trabalho no contexto comunitário, elas contribuem para um melhor conhecimento do sistema de saúde, o que facilitaria a apropriação do processo de trabalho e a aproximação da realidade das comunidades. Esse tipo de conhecimento favorece a resolubilidade das ações do PEF no SUS.

No mesmo sentido do pequeno número de respostas positivas ao contato com o tema "Saúde Pública/Saúde Coletiva” ao longo do curso de graduação, observouse que apenas 5\% dos PEF entrevistados realizaram estágio(s) supervisionado(s) na temática. Isso reforça a percepção dos PEF no que diz respeito à deficiência dos cursos em prepará-los para atuar no contexto coletivo.

Neste sentido, Freitas et al. ${ }^{16}$ relatam que o curso de graduação em Educação física não prioriza a integração entre ensino e trabalho. Este é um assunto que merece atenção, pois as Diretrizes Curriculares Nacionais incentivam a inserção de estudantes no SUS, o que segundo HADDAD et al. ${ }^{17}$, contribui para o conhecimento e compromisso com a realidade de saúde do país e região. Para Anjos e Duarte ${ }^{14}$, o estágio aproxima o estudante do trabalho, oportuniza a construçáo ativa do conhecimento e consolida o conteúdo proposto pelas disciplinas específicas de "Saúde Pública/Saúde Coletiva". Além disso, Costa et al. ${ }^{12}$ afirmam que tanto o oferecimento da disciplina quanto a realização do estágio na temática podem despertar o interesse dos alunos do curso de graduação em Educação Física por esta área de atuação.

Sobre o segundo principal achado da pesquisa, constatou-se que 36\% dos PEF fizeram algum tipo de pós-graduação no campo da saúde e apenas quatro PEF fizeram residência multiprofissional em saúde. Diante da importância da integraçáo entre ensino 
e serviço para formação do profissional de saúde, acredita-se que aumentar a oferta de residências multiprofissionais, possa aprimorar o trabalho do profissional no SUS. A criação de cursos específicos de residência multiprofissional para atuação na Atenção Primária à Saúde é recente no Brasil, mas acredita-se que este seja um dos principais caminhos para se melhorar a atuação dos PEF nos NASF.

O Ministério da Saúde, em parceria com o Ministério da Educação criou as residências multiprofissionais no campo da saúde a partir de 2005 (Lei no $11.129 / 2005)^{18}$, orientadas pelos princípios e diretrizes do SUS e que visam qualificar os profissionais da saúde para atuarem no serviço público de saúde, levando em consideração as necessidades e realidades locais e regionais.

Também, os cursos oferecem atividades teóricas e práticas que favorecem a integração entre ensino e serviço, sendo destinado às categorias que compóem a área da saúde (exceto a Medicina) como, por exemplo, a Educação Física ${ }^{19}$. Além das residências multiprofissionais, futuras propostas de mestrado profissional com fins na atuaçáo dos PEF nos NASF também poderiam contribuir para melhorar esta formação em nível de pós-graduação ${ }^{20}$.

Acredita-se que uma formação inicial universitária mais abrangente (i.e. que envolva conhecimentos e vivências práticas nos campos da epidemiologia, SUS, Estratégia de Saúde da Família, promoção em saúde e gestão em saúde) possa se reverter ao formado em uma melhor capacidade de planejamento e atuação, resultando, como consequência, na melhoria da percepçáo para atuação profissional em Saúde Pública/Coletiva.

A implantação dos NASF suscitou diferentes reflexôes no campo da saúde. Entre elas está a reflexão sobre a formação dos profissionais que atuam nestes núcleos. Para Costa et al. ${ }^{12}$, a formação inicial é fundamental para inserção do PEF e consolidação da Educação Física neste novo campo de intervenção. Ainda segundo os autores, esta formação inicial deve contribuir para a preparação de profissionais que tenham domínio de conhecimento em todas as suas possíveis áreas de atuação, além de trabalhar a criticidade e preparar profissionais capazes de compreender e colaborar com a transformação da realidade social na qual estejam envolvidos.

Com a constante transformação do mercado de trabalho na área da saúde, a nova concepção ampliada de saúde assumida pelo SUS e a inserção do PEF no SUS, observa-se a urgente necessidade de investigar e, se necessário, rediscutir a formação inicial e continuada em Educação Física para a atuação neste campo. Essa necessidade é evidenciada ao verificar que, de acordo com a opinião da maioria dos PEF entrevistados no presente estudo, os cursos de graduação não ofereceram disciplinas especificas relacionadas com o tema "Saúde Pública/Saúde Coletiva”, não ofereceram estágios específicos e não ofereceram uma formação adequada para atuação profissional no campo da saúde. Costa et al. ${ }^{12}$ relatam que algumas Instituiçóes de Ensino Superior (IES) estão buscando aprimorar seus conteúdos curriculares com o objetivo de oferecer uma formação adequada para a atuação do PEF no SUS.

Outra ação relevante para melhorar a formação para atuação profissional dos PEF em Saúde Pública é a participação nos Programas de Educação pelo Trabalho em Saúde (PET-Saúde), que visam reorientar a formação profissional por meio de vivências práticas em saúde ${ }^{21}$. Segundo Freitas et al. ${ }^{16}$, o PET-Saúde é um exemplo de experiências que procuram integrar formação e atuação profissional. Esse programa pressupóe a educação pelo trabalho e estimula à integração ensino-serviço-comunidade.

Além de todas estas ações, também podem promover o aprofundamento sobre o tema as ligas acadêmicas de atividade física e saúde, similar ao que já ocorre em outros campos da saúde como Medicina e Nutrição e o uso de metodologias ativas de aprendizagem, projetos e cursos de extensão aplicados às UBS, que por sua vez podem contribuir muito para melhorar a formação para a atuação dos PEF nos NASF ${ }^{20}$.

Para Costa et al. ${ }^{12}$, essas alterações ainda são insuficientes e torna-se necessária a continuidade dos estudos em pós-graduação para suprir tais deficiências. Sabe-se que diante da complexidade do tema em questão, não se pode esperar que apenas no curso de graduação seja realizado o aprofundamento suficiente dos conhecimentos relacionados à atuação do PEF no NASF. O curso de graduação deve oportunizar a reflexão sobre o assunto e na pós-graduação se dá a continuidade da preparação profissional.

Como limitação do presente estudo, devido à falta de instrumentos de coleta que atendessem as necessidades desta pesquisa, elaborou-se uma entrevista estruturada específica que, apesar de ter sido discutida amplamente por diversos pesquisadores especialistas na área e ter tido uma fase de pré-testes, a mesma foi aplicada pela primeira vez no presente trabalho. Portanto, sugere-se a aplicação desta ferramenta em PEF que atuam NASF situados em outras regiōes 
do país. Sugere-se, também, estudos qualitativos que possam aprofundar as questôes levantadas no presente estudo.

Dessa forma, conclui-se apenas uma pequena parcela dos PEF atuantes nos NASF do Estado de São Paulo teve um contato com o tema "Saúde Pública/Saúde Coletiva”, seja na forma de disciplinas ou em estágios supervisionados, o que caracteriza uma formação distanciada daquela que se pretende para a atuação neste contexto. Ainda no que se refere ao curso de graduaçáo, a maioria dos PEF avaliou que não teve uma boa formação para atuar no campo da Saúde Pública no NASF. Sugere-se que reflexôes e discussóes sobre possíveis melhoras na formação do curso de graduação e de pós-graduação dos PEF para atuarem nos NASF devem ser fomentadas por gestores, professores e associações profissionais que atuam nesta área.

\begin{abstract}
Education of Physical Education professionals of nucleus of support to family health of the State of São Paulo

The aim of this study was to describe aspects related to higher education of Physical Education professionals (PEF) active in Nucleus of Support to Family Health (NASF) of São Paulo. For this purpose, a descriptive cross-sectional survey was conducted through a structured telephone interview, in which data were collected on the formation of 91 PEFs linked to NASF. As a result, it was observed that only $22 \%$ of the PEF had contact with the theme "Public Health / Collective Health" in the undergraduate course $(n=20)$ and less than $5 \%$ had multiprofessional residency in health $(n=5)$. The majority of the professionals $(61.5 \%, n=56)$ disagreed that they had adequate training to work in the SUS, and this percentage of disagreement did not have major changes according to the age group, graduation time and whether or not Postgraduate course. The data presented show that the PEF acting in the NASF of the state of São Paulo reported that they had a distance formation from the one that is intended to perform in this context. Reflections and discussions about possible improvements in the graduation and postgraduate training of PEF to work in the NASF should be fostered by managers and teachers who work in this area.
\end{abstract}

KEYwords: National Health Care System; Physical Education; Vocational Training; Public Health.

\title{
Agradecimentos
}

Agradecemos a todos os Profissionais de Educação Física dos Núcleos de Apoio a Saúde da Família do Estado de São Paulo.

\section{Conflito de interesse}

Não há conflito de interesse.

\section{Referências}

1. Brasil. Conselho Nacional de Secretários de Saúde. Sistema Único de Saúde. Brasília: CONASS; 2011. (Para Entender a Gestão do SUS, 1).

2. Brasil. Ministério da Saúde. Secretaria de Atenção à Saúde. Departamento de Atenção Básica. Diretrizes do NASF: Núcleo de Apoio a Saúde da Família. Brasília: Ministério da Saúde; 2010. 
3. Brasil. Ministério da Saúde. Secretaria de Vigilância em Saúde. Política nacional de promoção da saúde. Brasília: Ministério da Saúde; 2006.

4. Buss PM. Uma introdução ao conceito de promoção da saúde. In: Czeresnia D, Freitas CM, organizadores. Promoção da saúde: conceitos, reflexóes, tendências. Rio de Janeiro: Editora Fiocruz; 2003. p. 15-38.

5. Brasil. Lei $n^{\circ}$ 12.864, de 24 de setembro de 2013: altera o caput do art. $3^{\circ}$ da Lei $n^{\circ}$ 8.080, de 19 de setembro de 1990, incluindo a atividade física como fator determinante e condicionante da saúde, e dá outras providências. Diário Oficial da União [Internet]. 25 set 2013 [citado 23 jul 2019];1:5. Disponível em: https:/www.planalto.gov.br/ccivil_03/_ato20112014/2013/lei/l12864.htm.

6. Florindo AA, Hallal PC, Moura EC, Malta DC. Practice of physical activities and associated factors in adults, Brazil, 2006. Rev Saúde Pública. 2009;43(supl 2):65-73.

7. Hallal PC, Knuth AG, Reis RS, et al. Time trends of physical activity in Brazil (2006-2009). Rev Bras Epidemiol. 2011;14(Supl 1):53-60.

8. Brasil. Ministério da Saúde. Portaria no 154, de 24 de janeiro de 2008: cria os Núcleos de Apoio à Saúde da Família - NASF. Diário Oficial da União [Internet]. 4 mar 2008 [citado 23 jul 2019];1:38-42. Disponível em: http://bvsms.saude.gov.br/bvs/ saudelegis/gm/2008/prt0154_24_01_2008.html.

9. Brasil. Ministério da Saúde. Portaria no 2.681, de 7 de novembro de 2013: redefine o Programa Academia da Saúde no âmbito do Sistema Único de Saúde (SUS). Diário Oficial da União [Internet]. 8 nov 2013 [citado 23 jul 2019];1:37-8. Disponível em: http://bvsms.saude.gov.br/bvs/saudelegis/gm/2013/prt2681_07_11_2013.html.

10. Florindo AA, Nakamura PM, Farias Júnior JC, et al. Promotion of physical activity, healthy eating and family health in municipalities with health gym. Rev Bras Educ Fís Esporte. 2016;30(4). No prelo.

11. Romero A, Gerra PH, Costa EF, Santos T, Andrade D, Florindo A. Interventions by Physical Education professionals in Family Health Support Units in São Paulo. Rev Bras Ativ Fís Saúde. 2016;21(1):55-66.

12. Costa LC, Lopes Junior CAF, Costa EC, Feitosa MC, Aguiar JB, Gurgel LA. Formação profissional e produtividade em Saúde Coletiva do Profissional de Educação Física. Rev Bras Ativ Fis Saúde. 2012;17(2):107-13.

13. Scabar TG. A educação física e a promoção da saúde: formação profissional e desenvolvimento de competências [dissertação]. São Paulo (SP): Universidade de São Paulo, Faculdade de Saúde Pública; 2014.

14. Anjos TC, Duarte ACGO. A educação física e a estratégia saúde da família: formação e atuação profissional. Rev Saúde Colet. 2009;19(4):1127-44.

15. Souza SC, Loch MR. Intervenção do profissional de Educação Física nos Núcleos de Apoio à Saúde da Família em municípios do norte do Paraná. Rev Bras Ativ Fis Saúde. 2011;16(1):5-10.

16. Freitas FF, Carvalho YM, Mendes VM. Educação Física e Saúde: aproximaçôes com a "clínica ampliada”. Rev Bras Ciênc Esporte. 2013;35(3):639-56.

17. Haddad AE, Morita MC, Pierantoni CR, Brenelli SL, Passarella T, Campos FE. Formaçáo de profissionais de saúde no Brasil: uma análise no período de 1991 a 2008. Rev Saúde Pública. 2010;44(3):383-93.

18. Brasil. Portaria Interministerial no 3.101, de 3 de novembro de 2005: institui o Programa Nacional de Reorientação da Formação Profissional em Saúde - Pró-Saúde. Brasília: Secretaria de Gestão do Trabalho e da Educação; 2005.

19. Loch MR, Florindo AA. A Educação Física e as residências multiprofissionais em saúde. Rev Bras Ativ Fis Saúde. 2012;17(1):81-2.

20. Andrade DR, Costa EF, Garcia LMT, Florindo AA. Formação do bacharel em educação física frente à situação de saúde no Brasil. In: Benedetti TRB, Silva DAS, Silva KS, Nascimento JV, organizadores. A formação do profissional de educação física para o setor Saúde. Florianópolis: Postmix; 2014. p. 87-107.

21. Brasil. Ministério da Saúde. Portaria Interministerial no 1.802, de 26 de agosto de 2008: institui o Programa de Educação pelo Trabalho para a Saúde - PET - Saúde [Internet]. Brasília: Ministério da Saúde; 2008 [citado 23 jul 2019]. Disponível em: http://bvsms.saude.gov.br/bvs/saudelegis/gm/2008/pri1802_26_08_2008.html.

ENDEREÇO

Alexandre Romero

Av. Arlindo Béttio, 1000 - Ermelino Matarazzo

São Paulo - São Paulo - Brasil

CEP: 03828-000

E-mail: aleromero@usp.br
Submetido: 26/01/2015

Revisado: 11/03/2017

Aceito: 29/06/2017 